\title{
Urethral duplication type influences on the complications rate and number of surgical procedures
}

Roberto Iglesias Lopes 1,2, Amilcar Martins Giron ${ }^{1}$, Marcos Figueiredo Mello 1, Cristovao Machado Barbosa Neto ${ }^{1}$, Joana dos Santos ${ }^{2}$, Paulo Renato Marcelo Moscardi ${ }^{1}$, Victor Srougi ${ }^{1}$, Francisco Tibor Denes $^{1}$, Miguel Srougi ${ }^{1}$

${ }^{1}$ Unidade de Urologia Pediátrica, Divisão de Urologia, Hospital das Clínicas, Faculdade de Medicina da Universidade de São Paulo, Brasil; ${ }^{2}$ Division of Urology, The Hospital for Sick Children, University of Toronto, Canada

\section{ABSTRACT}

Introduction: Urethral duplication is rare. Characterized by the presence of two urethral channels. This anomaly presents a great variety of clinical findings that depend on the type of duplication that often is associated with other anomalies.

Material and Methods: We report thirteen boys with urethral duplication managed in our institution between 1988-2015. Clinical findings, associated anomalies, treatment of urethral duplication and our results are described. Patients were classified according to Effmann classification.

Results: Mean patient's age was 38.3 \pm 34.7 months (3-136 months). Mean follow-up was $7.7 \pm 3.4$ years $(3 y 8 m-14 y 2 m)$. Type II A2 was the most common pattern $(8 / 13$ patients, 61.5\%), followed by type IA (3/13 patients, 23\%) and IIA1 (2/13 patients, 15.3\%). The most frequent clinical manifestations were urinary tract infections (UTI) observed in 11/13 patients (84.6\%) and anal urinary leakage, found in 7/13 patients (53.8\%). Associated anomalies were found in 9/13 patients (69.2\%).

Required surgeries were $3.53 \pm 2.84$ procedures per patient. Considering groups: Type IIA2 $4.25 \pm 3.28$, type IIA $1 \pm \pm 1.41$ and type IA $1.33 \pm 0.57$ needed procedures per patient. Complications rate were 0\% for type IA, 50\% for type IIA1 and 75\% for type IIA2.

Conclusions: Patients with incomplete duplication (type I A or I B) can totally be asymptomatic, with no need of surgical correction. Type IIA2 is the most complex form of duplication to correct and multiple procedures might be required because of the very hypoplastic orthotopic dorsal urethral tissue. Surgical treatment should be individualized and parents should be advised on complications and need of multiple surgeries according to urethral duplication type.

\section{ARTICLE INFO}

\section{Keywords:}

Surgical Procedures, Operative; Urethra; complications [Subheading]

Int Braz J Urol. 2017; 43: 1144-51

Submitted for publication:

May 21, 2016

Accepted after revision:

September 14, 2016

Published as Ahead of Print: February 03, 2017

\section{INTRODUCTION}

Urethral duplication is a rare congenital anomaly characterized by two urethral channels, whose location and extension present variations. It is more common in males, occurring usually in the sagittal plane. In females, this anomaly is rare and most often associated with bladder duplication (1-4).

Because of the diversity of clinical manifestations, diagnosis is difficult, as well as its classification. Patients can be either asymptomatic or symptomatic, most common clinical findings being incontinence, obstruction, recurrent urinary 
infection, and occasionally double urinary stream (1-4).

The objective of this study was to review our experience in the management of urethral duplication anomalies and to determine whether the type of urethral duplication influences on the number of surgical procedures needed for repair and complication rates.

\section{MATERIALS AND METHODS}

Medical records of patients with urethral duplication anomalies were analyzed retrospectively. We searched in our hospital database for urethral duplication cases submitted to surgical treatment. Urethral duplication cases without surgical management were not included in this study. Clinical characteristics such as age at presentation, type of urethral duplication, clinical findings, associated anomalies, surgical treatment, complications and results were reviewed. Patients were classified according to Effmann et al., as shown in Table-1 (5).

Thirteen male patients with urethral duplication were managed surgically at our institution between 1988 and 2016. All patients were carefully assessed preoperatively by clinical history, physical examination, kidney and bladder ultrasonography and voiding cystourethrography (VCUG). For surgical planning, an urethrocystoscopy was performed at the beginning of the operation to aid the surgical decision.

Uroflowmetry and urodynamics studies were only performed in cases of associated dysfunctional voiding (irritative or obstructive lower urinary tract symptoms). Follow-up was done by regular clinic visits (every 6 months to 1 year) that included physical examination and ultrasonography of urinary system. VCUG was carried out only if there was recurrent urinary tract infection or suspicion of urethral obstruction.

After collection of analytical data, urethral duplication type was correlated to the number of surgical procedures needed for repair and complications rates.

\section{Statistical analysis}

All values were presented as mean \pm standard deviation with the ranges. Statistical analysis was done using ANOVA (Bonferroni) for categorical comparisons. Results were considered significant when $\mathrm{p}$ value was equal or less than 0.05 .

Table 1 - Classification of urethral duplication (Effmann et al., 1976).

\begin{tabular}{|c|c|c|}
\hline Type I & & Incomplete urethral duplication (accessory urethra) \\
\hline & A - Distal: & $\begin{array}{l}\text { The meatus is on the dorsal or ventral surface, but it does not have } \\
\text { communication with the urethra or the bladder. }\end{array}$ \\
\hline & B - Proximal: & $\begin{array}{l}\text { The accessory urethra originates from the normal urethra } \\
\text { and ends blindly. }\end{array}$ \\
\hline \multirow[t]{6}{*}{ Type II } & & Complete Duplication \\
\hline & A - Two meatus: & \\
\hline & & $\begin{array}{l}1 \text { - two non-communicating urethras originating independently from } \\
\text { the bladder }\end{array}$ \\
\hline & & $\begin{array}{l}2 \text { - the second urethra originates from the first, with an independent } \\
\text { channel until the second meatus }\end{array}$ \\
\hline & B - One meatus: & \\
\hline & & $\begin{array}{c}1-\text { two urethras originate from the bladder or of the subsequent } \\
\text { urethra joining later in a single channel }\end{array}$ \\
\hline Type III & & Urethral duplication, part of a complete or incomplete caudal duplication \\
\hline
\end{tabular}




\section{RESULTS}

Mean age at surgical intervention was $38.3 \pm 34.7$ months (range: 3 to 136 months). Mean age \pm standard deviation (range) was $72.3 \pm 55.1$ (38 to 136 months) for group IA, $43 \pm 26.8$ (24 to 62 months) for group IIA1 and 24.3 \pm 19.3 (3 to 61 months) for group IIA2. Mean follow-up was $7.7 \pm 3.4$ years (3y8m-14y $2 \mathrm{~m})$.

Type IIA2 was the most common pattern, found in $8 / 13$ patients (61.5\%), followed by type IA (3/13 patients, 23\%) and IIA1 (2/13 patients, 15.3\%). The most frequent clinical manifestations were urinary tract infections (UTI) observed in $11 / 13$ patients $(84.6 \%)$ and anal urinary leakage, found in $7 / 13$ patients (53.8\%). Associated anomalies were found in 9/13 patients (69.2\%). The most common associated anomalies were vesicoureteral reflux (6/13 patients, $46 \%$ ) and renal abnormalities (4/13 patients, 30.7\%), as demonstrated in Table-2.

Required surgeries were $3.53 \pm 2.84$ procedures per patient ( 1 to 12 procedures). Considering groups: type IA needed $1.33 \pm 0.57$ procedures per patient ( 1 to 2 surgeries), type IIA 1 needed $4 \pm 1.41$ procedures per patient ( 3 to 5 surgeries) and type IIA2 need $4.25 \pm 3.28$ procedures per patient (2 to 12 surgeries). Children with types IIA1 and IIA2 of urethral duplication underwent significantly more surgical procedures than type IA ( $p$ values 0.05 and 0.05 , respectively). Complications rate were $0 \%$ for type IA, 50\% for type IIA1 (1/2 patients had urethral stenosis) and 75\% for type IIA2 (6/8 patients, with 6/8 developing urethral stenosis and 2/8 with a defunctionalized bladder that required augmentation). However, no statistically significant difference was found when number of surgeries for types IIA1 and IIA2 were compared $(p=1.0)$

\section{DISCUSSION}

Urethral duplication is a rare anomaly, with great diversity of clinical presentations. Some explanations of its morphogenesis have been proposed and as a consequence, several hypotheses were formulated aiming to explain failures in its embryogenesis.
The occurrence of urethral duplication with the accessory urethra epispadic, as in cases 2 and 3 can be associated with the same embryology theory of the exstrophy-epispadias complex, in which it might occur failure of the fusion of lateral mesoderm in the midline, between the ectoderm and the endoderm of the cloacal membrane (5).

In cases of duplication in which one of the urethral meatus is located in the anal or perineal region (cases 4 to 13), these are probably secondary to failure of the urorectal septum normal development (6).

In the type III urethral duplication, associated with the syndrome of caudal duplication, that can present duplications of the uterus, vagina, rectum, colon, among others, the morphogenetic defect occurs due to the division of the notochord in earlier phase of the embryonic development (5). In cases of collateral urethral duplication, that is, when both urethras are side by side, the urethral groove could be divided before forming the urethra in the medium line, originating two urethras (7).

In this study, Effmann et al. (5) classification was used because it is considered the most complete, as described in Table-1.

Patients' clinical findings with urethral duplication are variable, depending on the duplication type. According to Bogaert, (8) an accessory urethra ends blindly (type I A) and can cause few symptoms as elimination of purulent secretion or be asymptomatic. Type IB can also be asymptomatic, many times difficult of being differentiated of urethral diverticulum. In cases of duplication with epispadic accessory urethra, a dorsal curvature of the penis can occur; in the cases of hypospadic accessory urethra, ventral curvature can occur.

Types I B and IIB1 can be asymptomatic (8). Probably this is the reason why these are considered rare (difficult diagnosis due to the lack of clinical manifestations).

In cases of complete duplication, types IIA1 and IIA2, the most common clinical manifestations include recurrent urinary tract infections, double urinary stream and urinary obstruction. Type IIA2, with a perineal urethra or 


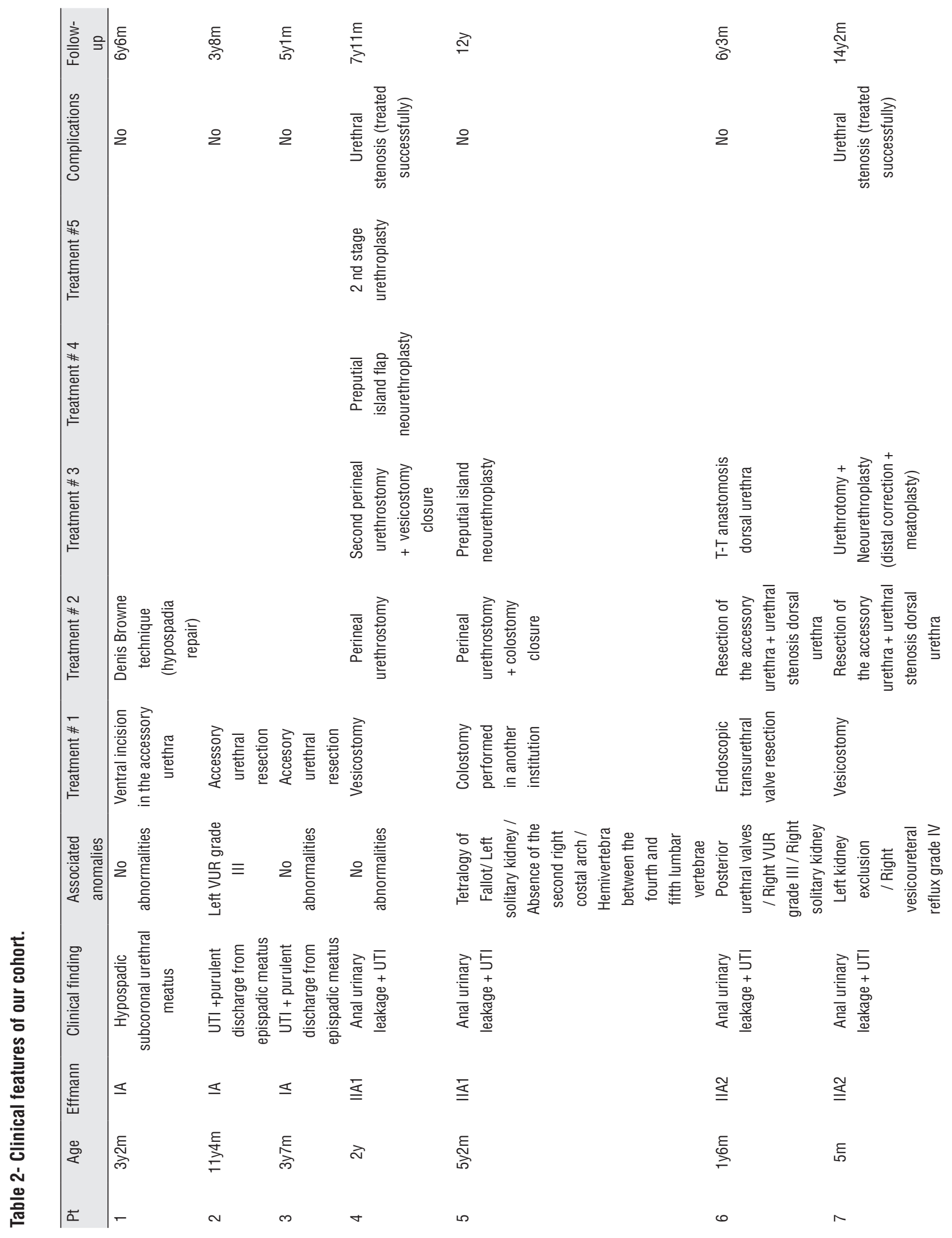


ริ

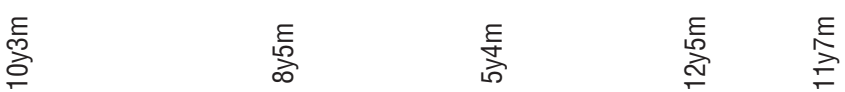

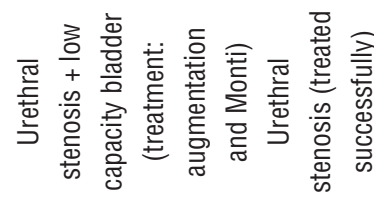

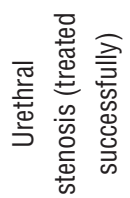

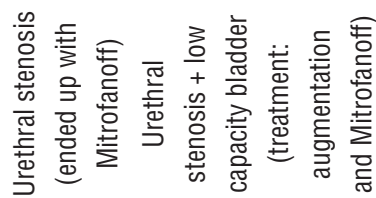

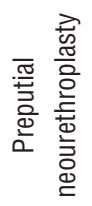

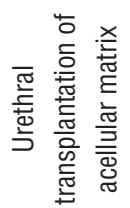

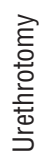

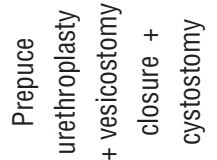

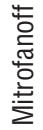

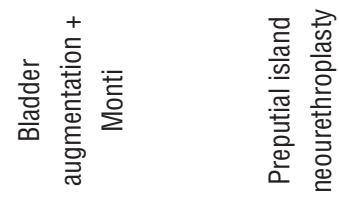

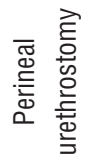

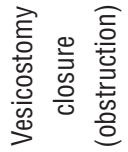

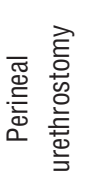

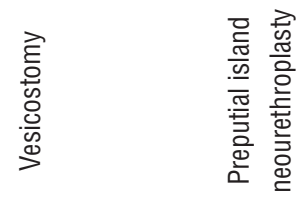

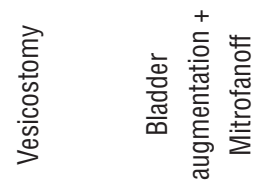

हे

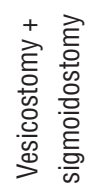

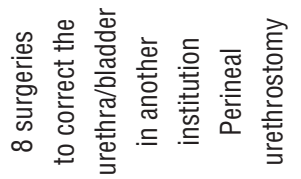

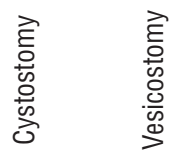

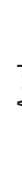

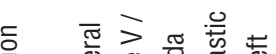

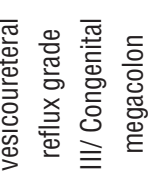

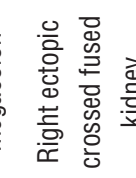

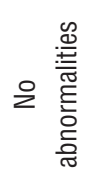

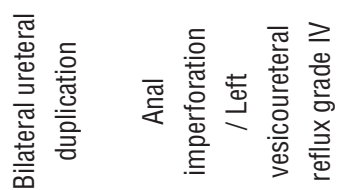

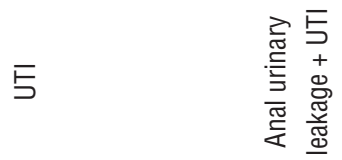

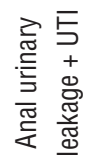

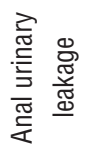

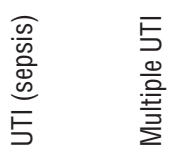

年

$\stackrel{\Upsilon}{=}$

$\widetilde{x}$

$\stackrel{\cong}{\cong} \quad \stackrel{\cong}{=}$

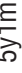

喜

$\underset{\mathrm{m}}{\stackrel{\mathrm{m}}{\mathrm{m}}}$

跒

E $\geq$

$\infty$

우 $\quad F$

$\stackrel{m}{\simeq}$ 
Figure 1- A) Voiding cystourethrogram revealed urethral duplication with a narrow cal-iber dorsal penile orthotopic urethra and the ventral urethra meatus opening in the rec-tum (type IIA urethral duplication); B) Catheterization of the ventral urethra by its opening in the rectum (type IIA urethral duplication); C) Intraoperative picture show-ing dissection of the ventral urethra; D) Intra-operative view depicting catheterization of the accessory urethral meatus located at penoscrotal junction; E) Onlay acellular matrix urethral transplantation; F) Final aspect of onlay acellular matrix urethral transplanta-tion (Patient 10 of Table-2).

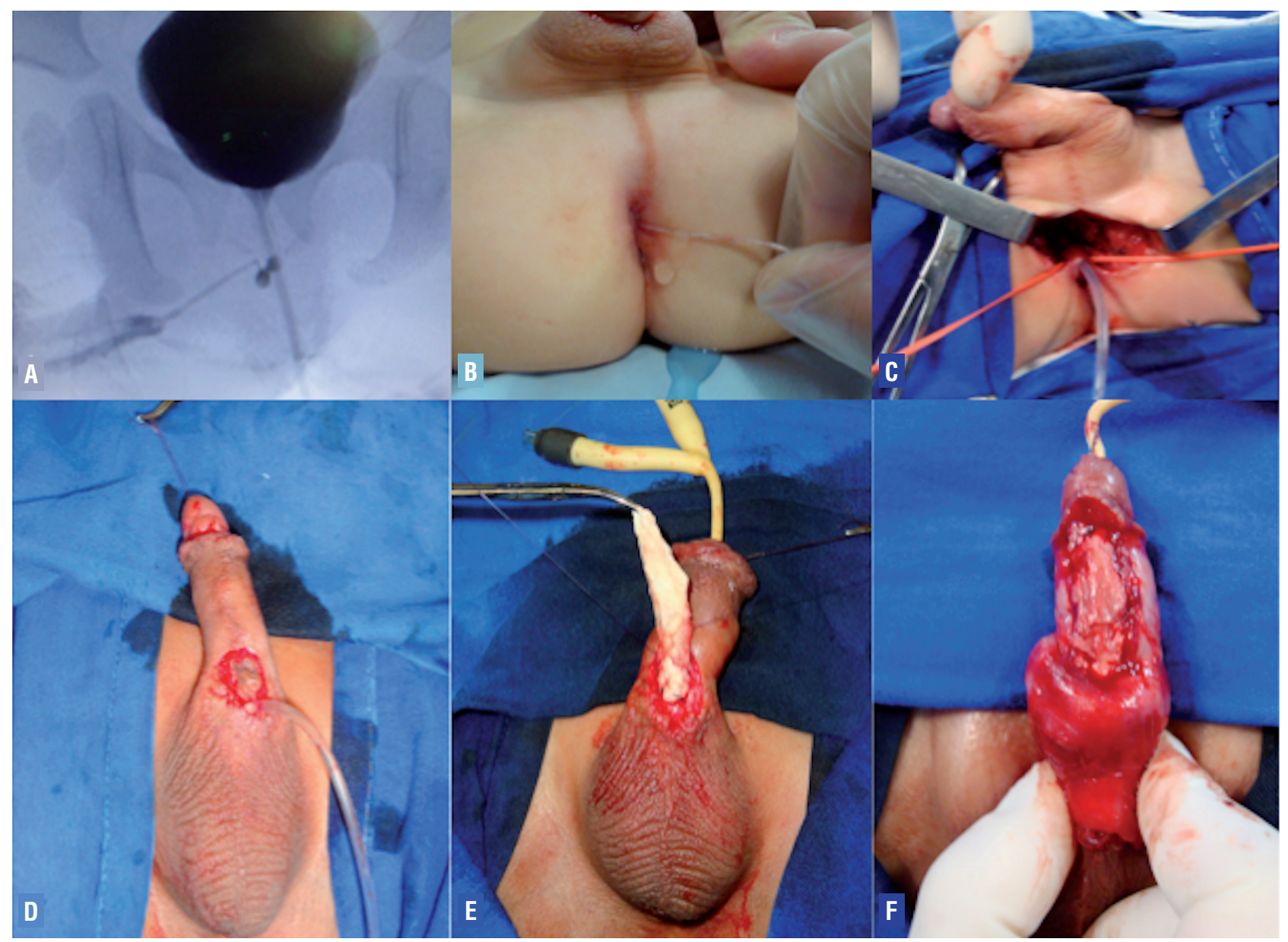

in the anal channel, also called duplication in $\mathrm{Y}$ or $\mathrm{H}$, usually presents a more functional ventral urethra. In those children, clinical findings may include urine elimination along with stool. However, in this presentation, some patients can present normal dorsal orthotopic urethra also called congenital urethroperineal fistula. It is considered as a separated entity of the urethral duplications by some authors.

Some patients with complete duplication can present urinary incontinence. Gross and Moore reported this manifestation in seven of 19 examined patients. In our cases, no patient presented urinary incontinence. In most of the cases, the ventral urethra crosses the sphincter, also containing the accessory glands and the verumontanum (3). These patients can also present stress urinary incontinence. This clinical manifestation is due to the accessory urethra, which is not usually surrounded by the urinary sphincter (9).

In cases of collateral duplication, the double flow is the most common clinical manifestation. These patients usually present several other associated congenital anomalies, such as colon and bladder duplications, hemivertebrae, dysplasia and renal agenesis, among others (9). 
No study about fertility and ejaculation in patients with urethral duplication was reported in the literature. The association between urethral duplication and posterior urethral valves was described by Lorenzo et al., (10) Fernbach et al. (11) and Ramanujam et al. (12). This association presents more difficulty to provide an accurate diagnosis of urethral duplications, as verified in case 6 .

Other anomalies associated with urethral duplication include bifid scrotum, cryptorchidism, hypospadias, megalourethra, micropenis, vesicoureteral reflux, agenesis and renal ectopy, dysplastic-multicystic kidney, vertebral anomalies (sacral agenesis, thoracic hemivertebra), anorectal anomalies, trachea-esophagic fistula and penile, vagina, uterus, bladder and colon duplications $(1,2,6,7,13)$.

The diagnosis of urethral duplication is performed by clinical history, physical exam and imaging methods, especially voiding cystourethrography. Kidney and bladder ultrasonography is recommended to investigate associated anomalies. Urethrocystoscopy is important for surgical planning. Magnetic resonance urography or excretory urography might be useful to further depict upper tract abnormalities.

Treatment of this anomaly depends on the duplication type and its clinical manifestations. Before any surgical decision, it must be identified which urethra is more functional. $\mathrm{Pa}-$ tients with incomplete duplication (type I A or I B) can totally be asymptomatic, with no need of surgical correction. If those patients present purulent secretions or local infection, the accessory urethra should be resected (8). Other option is the opening of the ventral wall of the accessory hypospadic urethra and posterior neourethroplasty, as described by Podesta et al. (14) to treat patients with hypospadias and incomplete urethral duplication. In these cases, it was observed fewer surgeries to proper surgical repair and no complications in our cohort.

In cases where the patient presents normal dorsal urethra and ventral urethra interfering in the anal canal (anorectal junction) or in the rectum (urethroperineal fistula) - type IIA2, our surgical approach aimed the initial urethros- tomy of the ventral urethra and preservation of the dorsal orthotopic urethra. After that, a neourethroplasty using flaps or grafts was usually performed. In difficult redo cases, even an acellular matrix transplantation was performed for one of our patients (case 10) as shown in Figure 1.

These patients with urethral duplication of hypospadic type, in which the ventral urethra is more functional and located in the perineal or anal area (type IIA2), are challenging to surgical correction and more commonly they present complications such as neourethral dehiscence and stenosis, which are common in these neourethroplasty types. In our study, a significant rate of surgical procedures per patient and complications were observed for type IIA duplications. Type IIA1 needed $4 \pm 1.41$ procedures per patient ( 3 to 5 surgeries) and type IIA2 need $4.25 \pm 3.28$ procedures per patient ( 2 to 12 surgeries) and rate of complications were 50\% for type IIA1 and 75\% for type IIA2 which should be informed for patients and families preoperatively. Type IIA2 is the most complex form of duplication to correct, and in such cases the orthotopic urethra usually has an extensive hypoplastic segment. Hence, it is recommended to mobilize extensively the ventral functional urethra to the perineal-scrotal junction to prevent complications and anticipate that multiple procedures might be required because of the very hypoplastic orthotopic dorsal urethral tissue.

\section{CONCLUSIONS}

Urethral duplication is a rare anomaly, with several forms of clinical presentation, often accompanied by other anomalies, and sometimes with difficult diagnosis. The treatment of urethral duplication should be individualized, according to its type. Significantly higher rates of surgical procedures per patient and, possibly complication rates were observed for type IIA duplications, which should be informed for patients and families preoperatively.

\section{CONFLICT OF INTEREST}

None declared. 


\section{Ethical approval}

The Institutional Review Board at Hospital das Clínicas da Faculdade de Medicina da Universidade de São Paulo approved this study.

\section{REFERENCES}

1. Prasad N, Vivekanandhan KG, Ilangovan G, Prabakaran S. Duplication of the urethra. Pediatr Surg Int. 1999;15:419-21.

2. Salle JL, Sibai H, Rosenstein D, Brzezinski AE, Corcos J. Urethral duplication in the male: review of 16 cases. J Urol. 2000;163:1936-40.

3. Onofre LS, Gomes AL, Leão JQ, Leão FG, Cruz TM, Carnevale J. Urethral duplication--a wide spectrum of anomalies. J Pediatr Urol. 2013;9:1064-71.

4. Coleman RA, Winkle DC, Borzi PA. Urethral duplication: cases of ventral and dorsal complete duplication and review of the literature. J Pediatr Urol. 2010;6:188-91.

5. Effmann EL, Lebowitz RL, Colodny AH. Duplication of the urethra. Radiology. 1976;119:179-85.

6. deVries PA, Friedland GW. Congenital "H-type" ano-urethral fistula. Radiology. 1974;113:397-407.

7. Kennedy HA, Steidle CP, Mitchell ME, Rink RC. Collateral urethral duplication in the frontal plane: a spectrum of cases. J Urol. 1988;139:332-4.

8. Bogaert GA. Urethral duplication and other urethral anomalies. In: Gearhart JP, Rink RC, Mouriquand PDE, editors. Pediatric urology, 1st ed., Philadelphia: WB Saunders; 2001; pp. 607-19.
9. Atala A. Congenital urethral duplication. In: Marshall FF, editor. Textbook of operative urology. Philadelphia: WB Saunders; 1996; pp. 992-1006.

10. Lorenzo RL, Turner WR, Bradford BF, Upshur J, Sexton FM. Duplication of the male urethra with posterior urethral valves. Pediatr Radiol. 1981;11:39-41.

11. Fernbach SK, Maizels M. Posterior urethral valves causing urinary retention in an infant with duplication of the urethra. J Urol. 1984;132:353-5.

12. Ramanujam TM, Sergius A, Usha V, Ramanathan S. Incomplete hypospadiac urethral duplication with posterior urethral valves. Pediatr Surg Int. 1998;14:134-7.

13. Savanelli A, Schiano A, Esposito C, Russo S, Dolezalova $\mathrm{H}$. Congenital megalourethra associated with urethral duplication and imperforate anus. Pediatr Surg Int. 1998;13:607-9.

14. Podesta ML, Medel R, Castera R, Ruarte AC. Urethral duplication in children: surgical treatment and results. J Urol. 1998;160:1830-3.

Correspondence address:

Marcos Figueiredo Mello, MD Divisão de Urologia, Hospital das Clínicas Faculdade de Medicina da Universidade de São Paulo Rua Dr. Éneas de Carvalho Aguiar, 455 - 7 andar

Telephone: + 5511 3069-8080 E-mail: marcosmello13@gmail.com 